OPEN ACCESS

Edited by:

Marco Scarpa

University Hospital of Padua, Italy

Reviewed by:

Xianjun $Y u$,

Fudan University, China Giovanni Battista Levi Sandri, San Camillo-Forlanini Hospital, Italy Giuseppe Portale,

Azienda ULSS 6 Euganea, Italy

*Correspondence:

Shengping $L i$

lishengp@mail.sysu.edu.cn

tThese authors have contributed equally to this work

Specialty section:

This article was submitted to Surgical Oncology,

a section of the journal

Frontiers in Oncology

Received: 12 April 2020

Accepted: 20 July 2020

Published: 03 September 2020

Citation:

He C, Sun S, Zhang Y, Lin X and Li S (2020) A Novel Nomogram to Predict

Survival in Patients With Recurrence of Pancreatic Ductal Adenocarcinoma

After Radical Resection

Front. Oncol. 10:1564.

doi: 10.3389/fonc.2020.01564

\section{A Novel Nomogram to Predict Survival in Patients With Recurrence of Pancreatic Ductal Adenocarcinoma After Radical Resection}

\author{
Chaobin $\mathrm{He}^{1 \dagger}$, Shuxin Sun ${ }^{1 \dagger}$, Yu Zhang ${ }^{2 \dagger}$, Xiaojun Lin ${ }^{1}$ and Shengping $\mathrm{Li}^{1 *}$ \\ ${ }^{1}$ State Key Laboratory of Oncology in South China, Department of Pancreatobiliary Surgery, Collaborative Innovation Center \\ for Cancer Medicine, Sun Yat-sen University Cancer Center, Guangzhou, China, ${ }^{2}$ State Key Laboratory of Ophthalmology, \\ Zhongshan Ophthalmic Center, Sun Yat-sen University, Guangzhou, China
}

The post-progression survival (PPS) of patients with pancreatic ductal adenocarcinoma (PDAC) after radical resection is varied and influenced by the characteristics of tumor progression. We aimed to establish and validate a nomogram to predict PPS for PDAC patients after surgery. A total of 302 PDAC patients who had undergone curative resection from 2008 to 2018 were enrolled in this study and randomly divided into training and validation cohorts at a ratio of 3:1. The nomogram was established based on independent prognostic factors selected by LASSO and Cox regression and measured by the area under the receiver operating characteristic curve (AUC) and the concordance index (C-index). Significant prognostic factors included carbohydrate antigen 19-9 (CA19-9), lymph node (LN)9 metastasis, LN14 metastasis, LN16 metastasis, tumor differentiation, imaging-detected tumor size, local progression, liver-only metastasis, lung-only metastasis, and multiple metastases. The nomogram built on these factors showed powerful efficacy in PPS prediction, with C-index values of 0.751 (95\% Cl $0.692-0.0 .810)$ and 0.710 (95\% Cl 0.645-0.755) for the training and validation cohorts, respectively. The AUC values for the 1-year and 2 -year PSS rates were $0.745,0.747$, and $0.783,0.748$, respectively; these values were higher than those of the 8th tumor-nodemetastasis (TNM) stage system. The exploration of risk factors and the establishment of a nomogram can provide new versions of personalized recurrence management for PDAC patients after surgery.

Keywords: pancreatic ductal adenocarcinoma, recurrence, surgery, nomogram, prognosis

\section{INTRODUCTION}

Pancreatic ductal adenocarcinoma (PDAC) is a lethal disease with a 5-year overall survival (OS) rate of only 7\% (1). Despite its low incidence, cancer-related deaths of PDAC patients rank fourth in the United States and continue to increase; thus, PDAC is expected to become the second-most common cause of cancer-related death by 2030 (2). Surgical resection, the only way of obtaining curative treatment of PDAC, is suitable for less than $20 \%$ of patients and improves the 5 -year OS rate to $20-30 \%$ (3). Moreover, up to $80 \%$ of PDAC patients suffer recurrence soon after curative 
resection (4). Therefore, early recurrence poses a major challenge for the long-term survival of PDAC patients after curative resection.

Several stage systems have been used to estimate the OS or progression-free survival of PDAC patients $(5,6)$. These instruments were constructed on the basis of variables limited to primary tumor features. However, PDAC patients with varied progression patterns may have different rates of postprogression survival (PPS), which is greatly impacted by features of progression rather than primary tumor features $(4,7)$. Therefore, previously developed predictive systems may be less effective for PPS estimation in PDAC patients after surgery. Considering the absence of a predictive model specifically designed for PPS estimation, it was necessary to build a clinical prognostic predictive system to estimate PPS as well as recurrence after surgery in individual PDAC patients.

In the present study, we established a prognostic nomogram to predict the PPS of PDAC patients after curative resection. We also conducted comparisons of the efficacy of predicting survival prediction between this nomogram and a tumor-nodemetastasis (TNM) system.

\section{MATERIALS AND METHODS}

\section{Patients}

Consecutive PDAC patients who had undergone radical resection from 2008 to 2018 at Sun Yat-sen University Cancer Center (SYSUCC) were included in this study. The exclusion criteria were as follows: (1) distant metastasis before surgery, (2) history of a second tumor, (3) follow-up period $<1$ year, (4) missing information from follow-up records, and (5) microscopic or macroscopic incomplete resection. The margin for radical resection was defined as $1.5-2 \mathrm{~mm}$, as in previous studies $(8,9)$. This study was approved by the Institutional Review Board of Sun Yat-sen University Cancer Center. All procedures involving human participants in the present study were performed in accordance with the ethical standards of institutional and/or national research committees as well as the 1964 Helsinki Declaration and its later amendments or similar ethical standards. Written informed consent was obtained from the patients prior to treatment.

\section{Data Collection}

Resectability was judged by a pancreatic multidisciplinary team based on radiological examination, including computed tomography (CT), magnetic resonance imaging (MRI), and positron emission tomography/CT (PET-CT). Specialized pancreatic surgeons performed all radical resections of PDAC. An experienced pancreatic pathologist carried out the pathological diagnosis and description of the specimens, including such characteristics as tumor size, tumor differentiation, lymph node (LN) metastasis, LN total number, LN positive number, satellite foci, macrovascular and microvascular invasion, lymph vessels, and perineural and adjacent organ invasion. LN ratio (LNR) was defined as the proportion of positive $\mathrm{LN}$ in the total examined LN. Additionally, the associated radiological and clinical variables described in our previous studies (7) were included in the present study. All blood test indexes were obtained at the time at which tumor progression was diagnosed. Previously described (10) inflammation-based indexes, including the neutrophil-tolymphocyte ratio (NLR), the platelet-to-lymphocyte ratio (PLR), the modified Glasgow Prognostic Score (mGPS), the prognostic nutritional index (PNI), the prognostic index (PI), and the systemic immune-inflammation index (SII), were analyzed as well.

\section{Recurrence Patterns}

Information regarding the timing and pattern of recurrence was obtained at regular follow-up, which consisted of regular chest and abdominal CT, carbohydrate antigen 19-9 (CA199) measurement, and carcinoembryonic antigen (CEA) measurement every 3 months after surgery. Additional imaging modalities, such as MRI and PET/CT, were selectively performed to determine patterns of recurrence. When imaging findings were consistent with recurrence, biopsy was rarely performed. Otherwise, biopsy was conducted to confirm tumor progression or metastases. Either radiological or histological evidence was required for the diagnosis of disease recurrence. The date of the last follow-up occurred at the end of May 2019. The first location of recurrence was used to describe the recurrence patterns, which were categorized as in the study by Groot et al. (4). The cutoff value differentiating early and late progression was defined as 1 year following surgery (11). The terms liver-only and lung-only metastases referred to isolated hepatic and lung recurrence, respectively. The term others referred to isolated recurrence in other less common areas. Local recurrence and isolated distant metastasis occurring simultaneously were classified as local + distant while the term multiple referred to multiple distant metastases.

\section{Survival Outcomes and Statistical Analysis}

Tumor progressions occurring within and beyond 1 year following surgery were classified as early and late progressions, respectively. Comparisons between the early and late progression groups were conducted for various clinical and pathological variables using chi-square analysis. The main survival outcome of this study was PPS, which was defined as the duration from the date of tumor progression to the date of death or the last date of follow-up. The Kaplan-Meier method was used to estimate survival. When the survival curves were not crossed, the survival differences were compared using a log-rank test. When the survival curves were crossed, the survival differences were further analyzed by landmark analysis. Multivariate analysis was adopted to determine significant prognosis factors based on the results of univariate analysis and the least absolute shrinkage and selection operator (LASSO) logistic regression model, which was used to explore the relationships between pathological and radiological variables and PPS. The area under the receiver operating characteristic (ROC) curves (AUCs) and concordance indexes (C-indexes) of the multimarker algorithms were calculated and compared with those of the TNM stage system. A two-tailed $P<0.05$ was considered statistically significant. All statistical analyses were conducted 
using SPSS software version 22 (SPSS Inc., Chicago, IL, USA) and $\mathrm{R}$ software version 3.6.1 ( $\mathrm{R}$ Development Core Team; http://www.r-project.org).

\section{RESULTS}

\section{Patients}

A total of 355 PDAC patients had received radical resection from 2008 to 2018 at SYSUCC. Fifty-three patients were excluded from this study according to the exclusion criteria, including microscopic or macroscopic incomplete resection (10 patients), history of a second tumor (12 patients), and missing information from follow-up records (31 patients). Ultimately, 302 patients were included in the present study. Each patient was followed up for more than 1 year and the median followup time was 24.7 months [95\% confidence interval (CI) 20.329.1 months]. During the follow-up period, a total of 173 (57.3\%) patients developed tumor progressions after surgery. Comparisons between the early and late progression groups for clinical, pathological, and radiological variables are shown in Table 1. All patients were randomly divided into training ( $n$ $=227)$ and validation $(n=75)$ cohorts in a 3:1 ratio for the establishment and validation of the nomogram.

\section{COMPARISONS OF CHARACTERISTICS BETWEEN EARLY AND LATE PROGRESSION GROUPS}

Apart from 129 patients who were free of tumor progression, 129 and 44 patients were included in the early and late tumor progression groups, respectively. As shown in Table 1, the distribution of clinical factors including age, gender, and inflammation-based indexes, was balanced between these three groups, while higher CA19-9 and CEA levels were positively associated with early tumor progression. In terms of pathological factors, patients in the early progression group were more likely to have LN metastases as well as large and poorly differentiated tumors. Significantly large proportions of patients in the early progression group had LN16 metastases, imagingdetected vascular invasion, and more advanced stages of TNM. Additionally, compared with patients in the late progression group, those in the early progression group were more likely to have liver metastases and local recurrence.

\section{Comparisons of PPS Stratified by Different Progression Patterns}

Overall, there were six different types of tumor progressions for PDAC patients after surgery. Liver-only metastasis was the most common progression type, followed by local recurrence, local and distant progression, and lung-only metastasis. Metastases at other sites and multiple metastases occupied a small proportion of tumor progressions. The median PPS for all patients was 13.53 months (95\% CI 11.24-15.83), and the 1-, 2-, and 3-year PPS rates were $55.9,26.4$, and $10.7 \%$, respectively. Patients with different progression patterns had varied survival rates. As shown in Figure 1, patients with local recurrence had the longest median
PPS of 15.93 months (95\% CI 11.07-25.03), followed by patients with lung-only metastasis (median PPS 14.7 months, 95\% CI 14.00-30.43) and liver-only metastasis (median PPS 12.6 months, 95\% CI 9.83-15.77). Landmark analysis was used to analyze survival differences when the survival curves were crossed. The comparisons of survival rates between local recurrence and other sites, between liver-only metastasis and multiple metastases, and between lung-only metastasis and multiple metastases revealed that the former had significantly higher survival rates $(P<$ $0.05)$ than the latter at 1 year following tumor progression (the landmark point for the survival analyses). Further, patients with local progression had significantly higher survival rates than those with multiple analyses, while survival rates were similar between the other comparison groups. Overall, multiple metastases corresponded with the poorest survival rates among these progression patterns.

\section{Prognostic Factors for PPS}

In order to investigate prognostic factors for PPS, a LASSOpenalized Cox regression analysis was performed based on 48 high-dimensional radiological and pathological data to further reduce the number of factors in the selected panel with the best predictive performance using the 10 -fold cross-validation (Figure 2). Nine variables were selected for PPS prediction by the LASSO-Cox regression model, including LN9 metastasis, LN14 metastasis, LN16 metastasis, local recurrence, liver metastasis, lung metastasis, multiple metastases, tumor differentiation, and imaging-detected tumor size. These predictors, alone with the associated clinical variables identified by univariate analysis, were incorporated in the multivariate analysis. Independent prognostic factors for PPS in PDAC patients following surgery included CA19-9 $(\mathrm{HR}=2.524,95 \%$ CI 1.002-6.359, $P=$ 0.050 ), LN9 metastasis ( $\mathrm{HR}=1.351,95 \% \mathrm{CI} 1.092-3.430, P=$ 0.042 ), LN14 metastasis $(\mathrm{HR}=1.304,95 \%$ CI $1.074-1.944, P$ $=0.042)$, LN16 metastasis $(\mathrm{HR}=2.785,95 \%$ CI 1.736-10.534, $P=0.031)$, tumor differentiation $(\mathrm{HR}=0.492$, 95\% CI $0.248-$ $0.974, P=0.042)$, imaging-detected tumor size $(\mathrm{HR}=1.579,95 \%$ CI 1.187-2.371, $P=0.043$ ), local progression ( $\mathrm{HR}=5.952,95 \%$ CI $1.869-18.868, P=0.003)$, liver-only metastasis $(\mathrm{HR}=6.452$, 95\% CI 1.919-21.739, $P=0.003)$, lung-only metastasis (HR = $4.405,95 \%$ CI 1.869-18.868, $P=0.046$ ), and multiple metastases $(\mathrm{HR}=3.578,95 \%$ CI 1.147-15.887, $P=0.042)$ (Table 2).

\section{Construction and Validation of Nomogram for PPS Prediction}

As shown in Figure 3, a specific nomogram was built based on independent prognostic factors for PPS. LN16 metastasis demonstrated the most prominent effect in PPS prediction, followed by local recurrence and liver-only metastasis. Calibration plots showed high agreement between predicted and actual survival in both training and validation cohorts (Figure 4). The C-indexes of the nomogram based on the training and validation cohorts were 0.751 (95\% CI $0.692-$ 0.0 .810 ) and 0.710 (95\% CI $0.645-0.755)$, respectively; these values were significantly higher than those of the 8th TNM stage system (Table 3 ). Comparisons of discriminatory capacity between the nomogram and the 8th TNM stage system were 
TABLE 1 | Clinicopathological characteristics of patients with PDAC.

\begin{tabular}{|c|c|c|c|c|c|c|c|c|c|c|c|c|c|}
\hline \multirow[t]{2}{*}{ Characteristics } & & \multicolumn{5}{|c|}{ Time to progression } & \multirow[t]{2}{*}{ Characteristics } & & \multicolumn{5}{|c|}{ Time to progression } \\
\hline & & Absence & $\begin{array}{c}\text { Early } \\
\text { progression }\end{array}$ & $\begin{array}{l}\text { Late } \\
\text { progression }\end{array}$ & $N$ & $P$ & & & Absence & $\begin{array}{c}\text { Early } \\
\text { progression }\end{array}$ & $\begin{array}{l}\text { Late } \\
\text { progression }\end{array}$ & $N$ & $P$ \\
\hline Whole cohort & & 129 & 129 & 44 & 302 & & $\begin{array}{l}\text { Macrovascular } \\
\text { invasion }\end{array}$ & Absence & 120 & 114 & 39 & 273 & 0.408 \\
\hline \multirow[t]{2}{*}{ Age } & $\leq 60$ years & 74 & 70 & 20 & 164 & 0.391 & & Presence & 9 & 15 & 5 & 29 & \\
\hline & $>60$ years & 55 & 59 & 24 & 138 & & $\begin{array}{l}\text { Microvascular } \\
\text { invasion }\end{array}$ & Absence & 87 & 85 & 34 & 206 & 0.364 \\
\hline \multirow[t]{2}{*}{ Gender } & Male & 53 & 46 & 20 & 119 & 0.453 & & Presence & 42 & 44 & 10 & 96 & \\
\hline & Female & 76 & 83 & 24 & 183 & & $\begin{array}{l}\text { Lymph vessel } \\
\text { invasion }\end{array}$ & Absence & 65 & 55 & 20 & 140 & 0.199 \\
\hline \multirow[t]{2}{*}{ Recurrence } & Absence & 129 & 24 & 21 & 174 & $<0.001$ & & Presence & 62 & 76 & 74 & 162 & \\
\hline & Presence & 0 & 105 & 23 & 128 & & Perineural invasion & Absence & 70 & 55 & 21 & 146 & 0.174 \\
\hline \multirow[t]{5}{*}{ TNM stage } & IA & 33 & 10 & 11 & 54 & 0.001 & & Presence & 59 & 74 & 23 & 156 & \\
\hline & IB & 36 & 25 & 13 & 74 & & $\begin{array}{l}\text { Adjacent organ } \\
\text { invasion }\end{array}$ & Absence & 119 & 112 & 39 & 270 & 0.361 \\
\hline & $\| \mathrm{A}$ & 11 & 20 & 4 & 35 & & & Presence & 10 & 17 & 5 & 32 & \\
\hline & $\| \mathrm{B}$ & 32 & 38 & 9 & 79 & & LNR & 0 & 83 & 61 & 29 & 173 & 0.036 \\
\hline & III & 17 & 36 & 7 & 60 & & & $0-0.16$ & 26 & 32 & 8 & 66 & \\
\hline \multirow{7}{*}{$\begin{array}{l}\text { Recurrence } \\
\text { patterns }\end{array}$} & Absence & 129 & 24 & 21 & 174 & $<0.001$ & & $>0.16$ & 20 & 36 & 7 & 63 & \\
\hline & Local & 0 & 29 & 10 & 39 & & Satellite foci & Absence & 123 & 120 & 44 & 287 & 0.180 \\
\hline & Liver-only & 0 & 43 & 6 & 49 & & & Presence & 6 & 9 & 0 & 15 & \\
\hline & Lung-only & 0 & 10 & 2 & 12 & & $\begin{array}{l}\text { Pancreatic } \\
\text { membrane } \\
\text { invasion }\end{array}$ & Absence & 81 & 74 & 28 & 183 & 0.608 \\
\hline & Other sites & 0 & 1 & 4 & 5 & & & Presence & 48 & 55 & 16 & 119 & \\
\hline & Local + distant & 0 & 13 & 1 & 14 & & $\mathrm{PI}$ & 0 & 93 & 78 & 28 & 199 & 0.310 \\
\hline & Multiple & 0 & 9 & 0 & 9 & & & 1 & 31 & 40 & 13 & 84 & \\
\hline \multirow[t]{2}{*}{ LN metastasis } & Absence & 83 & 61 & 30 & 174 & 0.007 & & 2 & 5 & 11 & 3 & 19 & \\
\hline & Presence & 46 & 68 & 14 & 128 & & $\begin{array}{l}\text { Imaging tumor size } \\
(\mathrm{cm})\end{array}$ & $\leq 2$ & 63 & 30 & 11 & 104 & $<0.001$ \\
\hline \multirow[t]{2}{*}{ LN5 metastasis } & Absence & 127 & 129 & 44 & 300 & & & $2-4$ & 45 & 68 & 28 & 141 & \\
\hline & Presence & 2 & 0 & 0 & 2 & & & $>4$ & 21 & 31 & 5 & 57 & \\
\hline \multirow[t]{2}{*}{ LN6 metastasis } & Absence & 126 & 128 & 44 & 298 & 0.391 & $\begin{array}{l}\text { Imaging LN } \\
\text { metastasis }\end{array}$ & Absence & 73 & 75 & 27 & 175 & 0.856 \\
\hline & Presence & 3 & 1 & 0 & 4 & & & Presence & 56 & 54 & 17 & 127 & \\
\hline \multirow[t]{2}{*}{ LN7 metastasis } & Absence & 128 & 126 & 42 & 296 & 0.283 & $\begin{array}{l}\text { Imaging vascular } \\
\text { invasion }\end{array}$ & Absence & 106 & 90 & 38 & 234 & 0.018 \\
\hline & Presence & 1 & 3 & 2 & 6 & & & Presence & 23 & 39 & 6 & 68 & \\
\hline
\end{tabular}


TABLE 1 | Continued

\begin{tabular}{|c|c|c|c|c|c|c|c|c|c|c|c|c|c|}
\hline \multirow[t]{2}{*}{ Characteristics } & & \multicolumn{5}{|c|}{ Time to progression } & \multirow[t]{2}{*}{ Characteristics } & & \multicolumn{5}{|c|}{ Time to progression } \\
\hline & & Absence & $\begin{array}{c}\text { Early } \\
\text { progression }\end{array}$ & $\begin{array}{l}\text { Late } \\
\text { progression }\end{array}$ & $N$ & $P$ & & & Absence & $\begin{array}{c}\text { Early } \\
\text { progression }\end{array}$ & $\begin{array}{l}\text { Late } \\
\text { progression }\end{array}$ & $N$ & $P$ \\
\hline \multirow[t]{2}{*}{ LN8 metastasis } & Absence & 126 & 126 & 42 & 294 & 0.698 & $\begin{array}{l}\text { Imaging LN size } \\
(\mathrm{cm})\end{array}$ & $\leq 0.5$ & 72 & 76 & 29 & 177 & 0.715 \\
\hline & Presence & 3 & 3 & 2 & 8 & & & $0.5-1$ & 30 & 28 & 6 & 64 & \\
\hline \multirow[t]{2}{*}{ LN9 metastasis } & Absence & 125 & 125 & 42 & 292 & 0.885 & & $>1$ & 27 & 25 & 9 & 61 & \\
\hline & Presence & 4 & 4 & 2 & 10 & & NLR & $\leq 3.32$ & 89 & 79 & 29 & 197 & 0.423 \\
\hline \multirow[t]{2}{*}{ LN10 metastasis } & Absence & 127 & 125 & 43 & 295 & 0.710 & & $>3.32$ & 40 & 50 & 15 & 105 & \\
\hline & Presence & 2 & 4 & 1 & 7 & & dNLR & $\leq 3.32$ & 39 & 42 & 19 & 100 & 0.284 \\
\hline \multirow[t]{2}{*}{ LN11 metastasis } & Absence & 126 & 124 & 44 & 294 & 0.367 & & $>3.32$ & 90 & 87 & 25 & 202 & \\
\hline & Presence & 3 & 5 & 0 & 8 & & PLR & $\leq 98.13$ & 17 & 13 & 6 & 36 & 0.692 \\
\hline \multirow[t]{2}{*}{ LN12 metastasis } & Absence & 116 & 111 & 41 & 268 & 0.370 & & $>98.13$ & 112 & 116 & 38 & 266 & \\
\hline & Presence & 13 & 18 & 3 & 34 & & PNI & 0 & 31 & 26 & 8 & 65 & 0.633 \\
\hline \multirow[t]{2}{*}{ LN13 metastasis } & Absence & 103 & 92 & 36 & 231 & 0.181 & & 1 & 98 & 103 & 36 & 237 & \\
\hline & Presence & 26 & 37 & 8 & 71 & & SII & $\leq 1000$ & 90 & 86 & 30 & 206 & 0.867 \\
\hline \multirow[t]{2}{*}{ LN14 metastasis } & Absence & 122 & 117 & 42 & 281 & 0.375 & & $>1000$ & 39 & 43 & 14 & 96 & \\
\hline & Presence & 7 & 12 & 2 & 21 & & mGPS & 0 & 93 & 81 & 28 & 202 & 0.558 \\
\hline \multirow[t]{2}{*}{ LN15 metastasis } & Absence & 127 & 123 & 44 & 294 & 0.367 & & 1 & 23 & 33 & 11 & 67 & \\
\hline & Presence & 2 & 6 & 0 & 8 & & & 2 & 13 & 15 & 5 & 33 & \\
\hline \multirow[t]{2}{*}{ LN16 metastasis } & Absence & 127 & 113 & 44 & 284 & $<0.001$ & WBC & $\leq 10$ & 124 & 115 & 41 & 280 & 0.097 \\
\hline & Presence & 2 & 16 & 0 & 18 & & & $>10$ & 5 & 14 & 3 & 22 & \\
\hline \multirow[t]{2}{*}{ LN17 metastasis } & Absence & 124 & 125 & 44 & 293 & 0.424 & ALB (g/L) & $\leq 35$ & 19 & 21 & 6 & 46 & 0.895 \\
\hline & Presence & 5 & 4 & 0 & 9 & & & $>35$ & 110 & 108 & 38 & 256 & \\
\hline \multirow[t]{2}{*}{ LN18 metastasis } & Absence & 126 & 126 & 44 & 296 & 0.593 & CRP (ng/L) & $\leq 3$ & 93 & 81 & 28 & 202 & 0.251 \\
\hline & Presence & 3 & 3 & 0 & 6 & & & $>3$ & 36 & 48 & 16 & 100 & \\
\hline \multirow{3}{*}{$\begin{array}{l}\text { Positive LN } \\
\text { number }\end{array}$} & 0 & 83 & 61 & 29 & 173 & 0.016 & CA19-9 (U/ml) & $\leq 35$ & 34 & 16 & 9 & 59 & 0.018 \\
\hline & $1-3$ & 36 & 46 & 13 & 95 & & & $>35$ & 95 & 113 & 35 & 243 & \\
\hline & $>4$ & 10 & 22 & 2 & 34 & & CEA (ng/ml) & $\leq 5$ & 97 & 77 & 31 & 205 & 0.026 \\
\hline \multirow[t]{3}{*}{ Tumor size $(\mathrm{cm})$} & $\leq 2$ & 48 & 24 & 16 & 88 & 0.007 & & $>5$ & 32 & 52 & 13 & 97 & \\
\hline & $2-4$ & 60 & 68 & 18 & 146 & & HBV infection & Absence & 120 & 122 & 41 & 283 & 0.866 \\
\hline & $>4$ & 21 & 37 & 10 & 68 & & & Presence & 9 & 7 & 3 & 19 & \\
\hline \multirow{3}{*}{$\begin{array}{l}\text { Tumor } \\
\text { differentiation }\end{array}$} & Well & 0 & 2 & 0 & 2 & 0.009 & Chemotherapy & No & 78 & 58 & 24 & 160 & 0.043 \\
\hline & Moderate & 72 & 55 & 26 & 153 & & & Yes & 51 & 71 & 20 & 142 & \\
\hline & Poor & 57 & 72 & 18 & 147 & & & & & & & & \\
\hline
\end{tabular}

M, month; LN, Iymph node metastasis; LNR, Iymph node ratio; TNM, tumor-node-metastasis stage; PI, prognostic index; NLR, neutrophil-to-lymphocyte ratio; PLR, platelet-to-lymphocyte ratio; PNI, prognostic nutritional index; SII, systemic immune-inflammation index; mGPS, modified Glasgow Prognostic Score; WBC, white blood cell count; ALB, albumin; CRP, C-reactive protein; CA19-9, carbohydrate antigen 19-9; CEA, carcinoembryonic antigen; HBV, hepatitis B virus. 


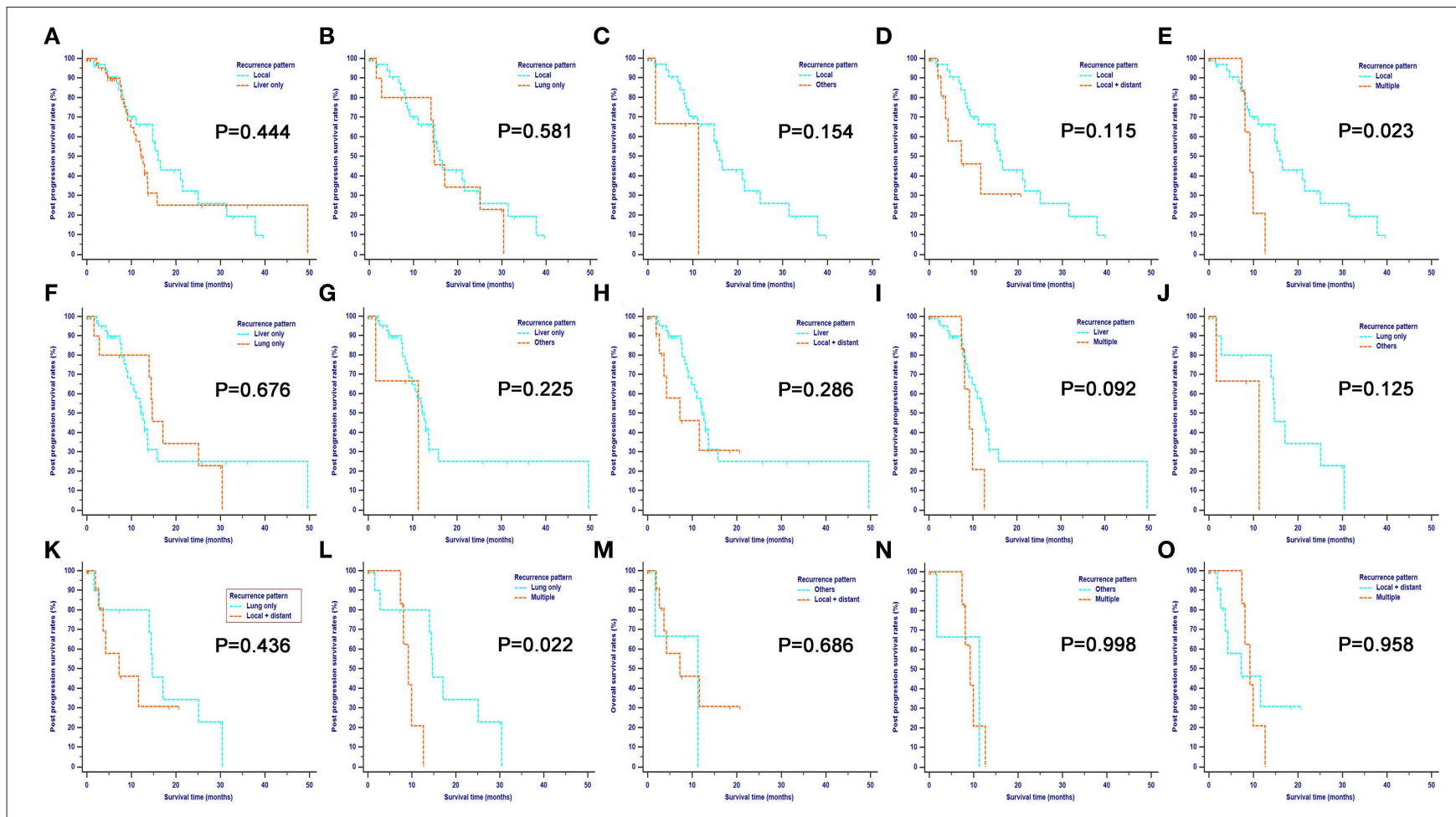

FIGURE 1 | Pairwise comparison of post-progression survival for different tumor progression patterns. Stratification of patients by comparing the following patterns of progression: local vs. liver only (A); local vs. lung only (B); local vs. others (C); local vs. local + distant (D); local vs. multiple (E); liver only vs. lung only (F); liver only vs. others (G); liver only vs. local + distant (H); liver only vs. multiple (I); lung only vs. others (J); lung only vs. local + distant (K); lung only vs. multiple (L); others vs. local + distant (M); others vs. multiple (N) and local + distant vs. multiple (0). Landmark analysis was used to analyze survival differences whose survival curves were crossed. For the comparisons of survival rates between local recurrence and other sites, liver-only metastasis and multiple metastases, lung-only metastasis, and multiple metastases, the former had significantly higher survival rates $(P<0.05)$, compared with the latter after 1 year since tumor progression, which was used as the landmark point for survival analyses. Also, patients with local progression had significantly higher survival rates compared with those with multiple analyses while survival rates were similar between other comparison groups. Overall, multiple metastases contributed to the poorest survival among these progression patterns.
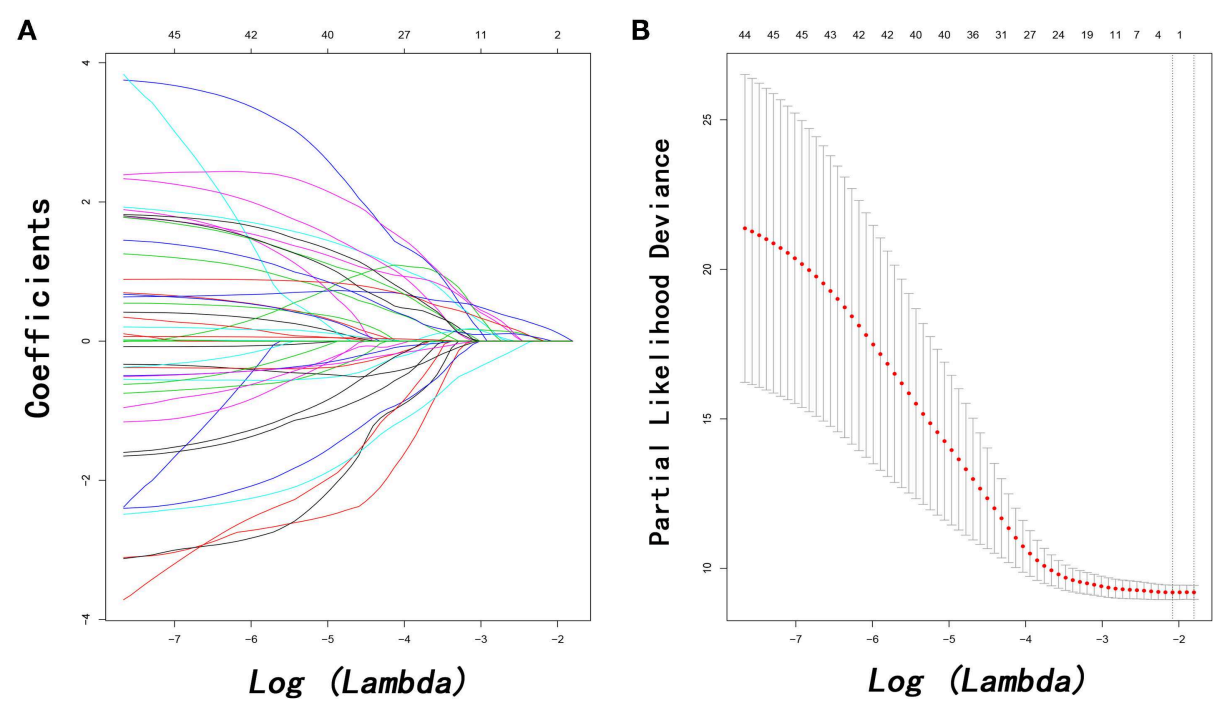

FIGURE 2 | Feature selection using the least absolute shrinkage and selection operator (LASSO) Cox regression model. LASSO coefficient profiles of 48 variables against the log (Lambda) sequence for PPS (A) and tuning parameter (Lambda) selection in the LASSO model used 10-fold cross-validation via minimum criteria for PPS (B). PPS, post-progression survival. 
TABLE 2 | Independent prognostic factors for PPS.

\begin{tabular}{|c|c|c|c|c|c|c|c|c|}
\hline \multirow[t]{2}{*}{ Characteristics } & \multicolumn{2}{|c|}{ Levels } & \multicolumn{6}{|c|}{ PPS } \\
\hline & & & HR & $95 \% \mathrm{Cl}$ & $\boldsymbol{P}$ & HR & $95 \% \mathrm{Cl}$ & $\boldsymbol{P}$ \\
\hline \multirow[t]{2}{*}{ Age } & $\leq 60$ years & 123 & Reference & & 0.819 & & & $\mathrm{NI}$ \\
\hline & $>60$ years & 104 & 0.942 & $0.563-1.576$ & & & & \\
\hline Gender & Male & 90 & Reference & & 0.088 & & & $\mathrm{NI}$ \\
\hline \multirow[t]{2}{*}{ WBC } & $\leq 10$ & 210 & Reference & & 0.010 & Reference & & 0.054 \\
\hline & $>10$ & 17 & 2.488 & $1.242-4.983$ & & 6.125 & $0.967-38.805$ & \\
\hline \multirow[t]{2}{*}{ NLR } & $\leq 3.32$ & 62 & Reference & & 0.527 & & & $\mathrm{NI}$ \\
\hline & $>3.32$ & 165 & 1.193 & $0.690-2.063$ & & & & \\
\hline dNLR & $\leq 3.32$ & 76 & Reference & & 0.215 & & & $\mathrm{NI}$ \\
\hline $\mathrm{PNI}$ & 1 & 178 & 1.277 & $0.647-2.522$ & & & & \\
\hline \multirow[t]{2}{*}{ SII } & $\leq 1000$ & 155 & Reference & & 0.173 & & & $\mathrm{NI}$ \\
\hline & $>1000$ & 72 & 1.505 & $0.836-2.709$ & & & & \\
\hline \multirow[t]{3}{*}{ mGPS } & 0 & 152 & Reference & & & & & $\mathrm{NI}$ \\
\hline & 1 & 50 & 1.072 & $0.501-2.296$ & 0.857 & & & \\
\hline & 2 & 25 & 1.198 & $0.494-2.909$ & 0.689 & & & \\
\hline \multirow[t]{3}{*}{ PI } & 0 & 149 & Reference & & & Reference & & \\
\hline & 1 & 64 & 0.435 & $0.201-0.944$ & 0.035 & 3.090 & $0.424-22.525$ & 0.266 \\
\hline & 2 & 14 & 0.384 & $0.161-0.920$ & 0.032 & 2.863 & $0.447-18.341$ & 0.267 \\
\hline ALB (g/L) & $\leq 35$ & 35 & Reference & & 0.815 & & & $\mathrm{Nl}$ \\
\hline \multirow[t]{2}{*}{ HBV infection } & Absence & 213 & Reference & & 0.445 & & & $\mathrm{Nl}$ \\
\hline & Presence & 14 & 1.577 & $0.490-5.080$ & & & & \\
\hline Chemotherapy & No & 120 & Reference & & 0.584 & & & $\mathrm{NI}$ \\
\hline & Yes & 107 & 1.165 & $0.675-2.010$ & & & & \\
\hline Time period to recurrence & $>24$ & 14 & Reference & & & & & $\mathrm{Nl}$ \\
\hline (month) & $\leq 6$ & 54 & 4.085 & $0.864-19.308$ & 0.076 & & & \\
\hline & $6-12$ & 43 & 3.244 & $0.766-13.748$ & 0.110 & & & \\
\hline & $12-24$ & 20 & 2.405 & $0.569-10.171$ & 0.233 & & & \\
\hline LN9 metastasis & Absence & 219 & & & & Reference & & 0.042 \\
\hline & Presence & 8 & & & & 1.351 & $1.092-3.430$ & \\
\hline LN14 metastasis & Absence & 211 & & & & Reference & & 0.038 \\
\hline & Presence & 16 & & & & 1.304 & $1.074-1.944$ & \\
\hline LN16 metastasis & Absence & 213 & & & & Reference & & 0.031 \\
\hline & Presence & 14 & & & & 2.785 & $1.736-10.534$ & \\
\hline Tumor differentiation & Well & 2 & & & & Reference & & \\
\hline & Moderate & 115 & & & & 0.569 & $0.051-6.305$ & 0.646 \\
\hline & Poor & 110 & & & & 0.492 & $0.248-0.974$ & 0.042 \\
\hline
\end{tabular}


TABLE 2 | Continued

\begin{tabular}{|c|c|c|c|c|c|c|c|c|}
\hline \multirow[t]{3}{*}{ Characteristics } & \multicolumn{2}{|c|}{ Levels } & \multicolumn{6}{|c|}{ PPS } \\
\hline & & & \multicolumn{3}{|c|}{ Univariate analysis } & \multicolumn{3}{|c|}{ Multivariate analysis } \\
\hline & & & HR & $95 \% \mathrm{Cl}$ & $\boldsymbol{P}$ & HR & $95 \% \mathrm{Cl}$ & $\boldsymbol{P}$ \\
\hline \multirow[t]{3}{*}{ Pathological tumor size (cm) } & $\leq 2$ & 66 & & & & Reference & & \\
\hline & $2-4$ & 110 & & & & 2.058 & $0.608-6.960$ & 0.246 \\
\hline & $>4$ & 51 & & & & 1.097 & $0.370-3.251$ & 0.867 \\
\hline \multirow[t]{3}{*}{ Imaging tumor size (cm) } & $\leq 2$ & 78 & & & & Reference & & \\
\hline & $2-4$ & 106 & & & & 1.579 & $1.187-2.371$ & 0.043 \\
\hline & $>4$ & 43 & & & & 0.840 & $0.461-1.531$ & 0.569 \\
\hline \multirow[t]{2}{*}{ Local progression } & Absence & 30 & & & & Reference & & 0.003 \\
\hline & Presence & 197 & & & & 5.952 & $1.869-18.868$ & \\
\hline \multirow[t]{2}{*}{ Liver-only metastasis } & Absence & 37 & & & & Reference & & 0.003 \\
\hline & Presence & 190 & & & & 6.452 & $1.919-21.739$ & \\
\hline \multirow[t]{2}{*}{ Lung-only metastasis } & Absence & 9 & & & & Reference & & 0.046 \\
\hline & Presence & 218 & & & & 4.405 & $1.869-18.868$ & \\
\hline \multirow[t]{2}{*}{ Other metastases } & Absence & 4 & & & & Reference & & 0.583 \\
\hline & Presence & 223 & & & & 0.590 & $0.090-3.872$ & \\
\hline \multirow[t]{2}{*}{ Local + distant metastasis } & Absence & 11 & & & & Reference & & 0.377 \\
\hline & Presence & 216 & & & & 0.516 & $0.119-2.240$ & \\
\hline \multirow[t]{2}{*}{ Multiple metastases } & Absence & 7 & & & & Reference & & 0.042 \\
\hline & Presence & 220 & & & & 3.578 & $1.147-15.887$ & \\
\hline \multirow[t]{2}{*}{ Microvascular invasion } & Absence & 155 & & & & Reference & & 0.533 \\
\hline & Presence & 72 & & & & 1.237 & $0.634-2.416$ & \\
\hline \multirow[t]{2}{*}{ Imaging vascular invasion } & Absence & 176 & & & & Reference & & 0.255 \\
\hline & Presence & 51 & & & & 0.519 & $0.195-1.542$ & \\
\hline \multirow[t]{3}{*}{ Imaging LN size (cm) } & $\leq 0.5$ & 133 & & & & Reference & & \\
\hline & $0.5-1$ & 48 & & & & 0.566 & $0.258-1.242$ & 0.156 \\
\hline & $>1$ & 46 & & & & 0.914 & $0.360-2.325$ & 0.851 \\
\hline \multirow[t]{3}{*}{ LNR } & 0 & 130 & & & & Reference & & \\
\hline & $0-0.16$ & 50 & & & & 0.502 & $0.223-1.130$ & 0.096 \\
\hline & $>0.16$ & 47 & & & & 0.447 & $0.185-1.080$ & 0.074 \\
\hline
\end{tabular}

PPS, post-progression survival; HR, hazard ratio; Cl, confidence interval; NI, not include; other abbreviations as in Table 1.

conducted using ROC curves (Figure 5). For the training and validation cohorts, the AUC values for 1-year and 2-year PSS rates were $0.745,0.747$, and $0.783,0.748$, respectively; these values were also higher than those of the 8th TNM stage system (Figure 5).

\section{DISCUSSION}

Recurrence is an important feature of PDAC after surgery, as it contributes to poor prognosis (7). Previous studies have shown that more than $60 \%$ of PDAC patients develop tumor recurrence (12). Similar results were also obtained in the present study, in that the patients in this study experienced a recurrence rate of $57.3 \%$. Given that the survival time of PDAC patients decreases significantly after tumor progression, it is necessary to establish an efficient prognostic system to predict PPS in these patients. Using a large cohort, we developed and validated a novel nomogram based on the characteristics of recurrence, which could be used to accurately stratify patients into distinct prognostic subgroups with significantly different PPS rates.

To date, many studies have consolidated that PDAC is a systemic disease $(4,11)$. Similar results were also obtained in the present study. In this study, most progressions occurred at the first year following surgery, indicating the systemic nature of this disease. Therefore, exploring the timing and patterns of recurrences is important in survival analyses of PDAC. Additionally, compared with OS, PPS was more influenced by recurrence-related factors as opposed to the characteristics of the primary surgery (13). In this study, most included prognostic factors were related to recurrence. Three additional variablesCA19-9, tumor size, and tumor differentiation-were found to be related to primary tumor status, suggesting that these factors have value for PPS estimation in addition to the effects on tumor recurrence.

Similar to previous studies $(4,7)$, the present study recorded six different types of recurrence patterns. Liver-only metastasis and local recurrence contributed to the majority of tumor 
Points

CA19-9 (U/ml)

LN16 metastasis

LN14 metastasis

LN9 metastasis

Local recurrence

Liver metastasis

Lung metastasis

Multiple metastases

Tumor differentiation

\section{Absence}

Absence

Absence

Absence

Absence

Presence

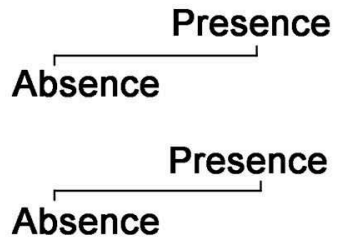

Presence

\section{Presence}

Presence

\section{Presence}

\section{Moderate}

Well Poor

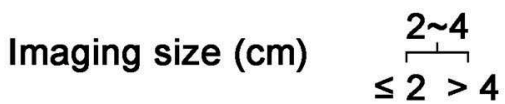

Total points

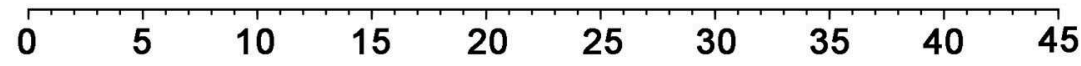

1-year PPS probability

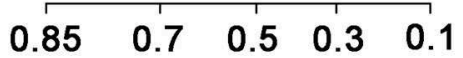

2-year PPS probability

\section{$\begin{array}{llll}0.7 & 0.5 & 0.3 & 0.1\end{array}$}

FIGURE 3 | Nomogram for predicting the 1- and 2-year post-progression survival rates in patients with post-operative recurrence of PDAC. PDAC, pancreatic ductal adenocarcinoma.

progressions, with most occurring in the early phase. Multiple metastases and distant metastases at sites apart from liver and lungs contributed to only a small proportion of tumor progressions. However, the presence of multiple metastases indicated the poorest PPS for PDAC patients compared with other types of tumor progressions. Patients with local recurrence had the longest median PPS, followed by patients with lungonly and liver-only metastases. Compared with other types 
A

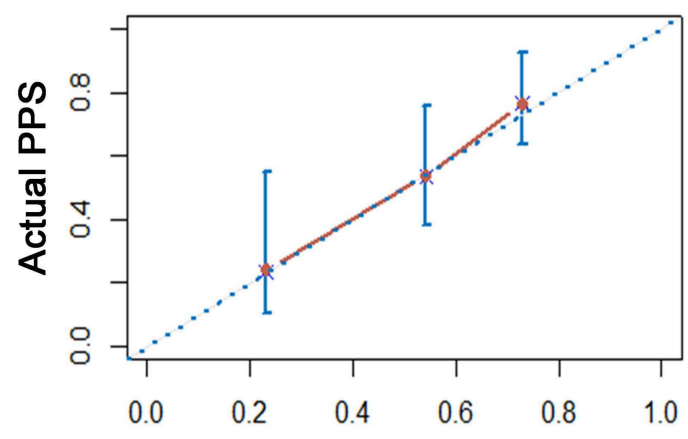

Nomogram-predicted 1-year PPS

C

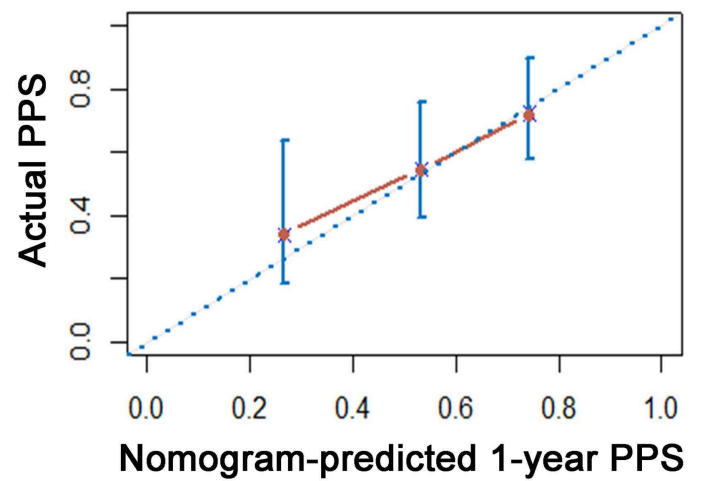

B

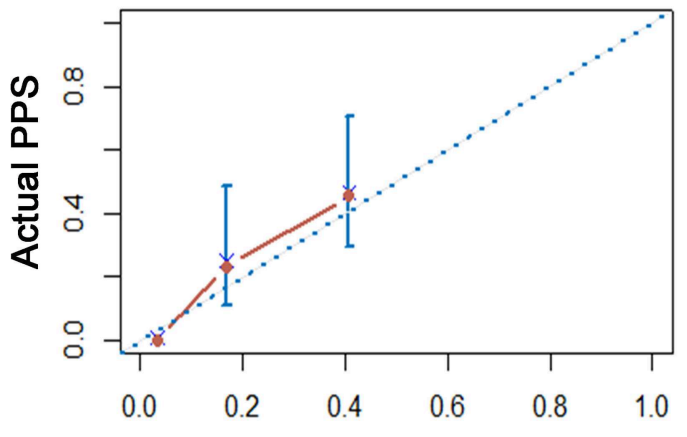

Nomogram-predicted 2-year PPS

D

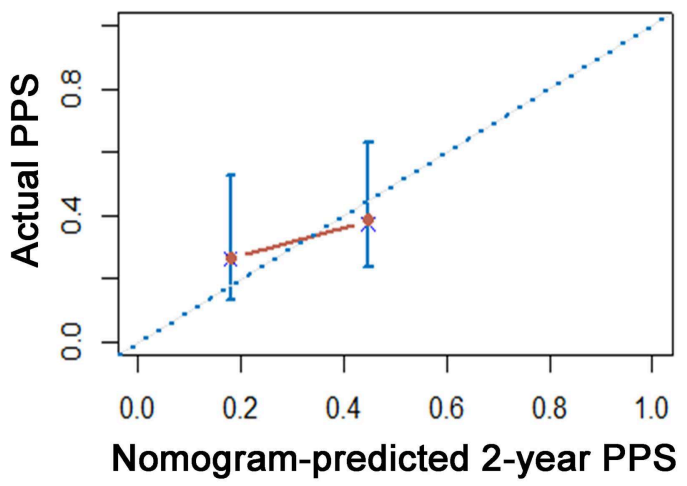

FIGURE 4 | The calibration curve for predicting patient survival at 1 year and 2 years in the training cohort $(\mathbf{A}, \mathbf{B})$ and validation cohorts $(\mathbf{C , D})$, respectively.

TABLE 3 | Comparison of the C-index and AUC values between nomograms and TNM stage.

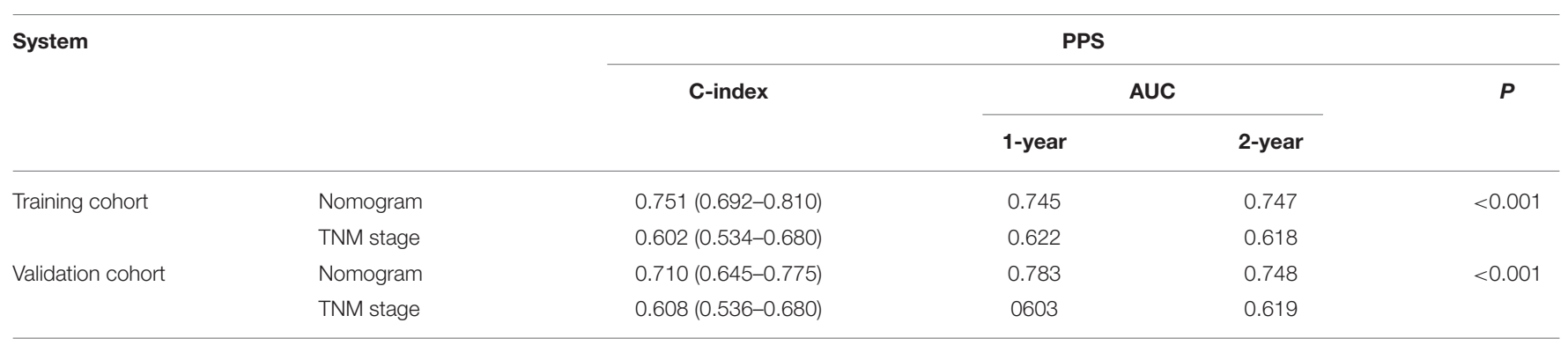

PPS, post-progression survival; TNM, tumor-node-metastasis; AUC, area under receiver operating characteristic curve; C-index, concordance index; other abbreviations as in Table 1.

of tumor progressions, largeness of the tumor bed capacity and the functional preservation of the lungs or liver in lung or liver metastases were helpful for obtaining longer survival times after tumor progressions. Moreover, lung-only and liveronly metastases shared similar survival rates. A 48 highdimensional radiological and pathological data was incorporated into the LASSO regression, showing that LN9 metastasis, LN14 metastasis, LN16 metastasis, tumor differentiation, imagingdetected tumor size, local progression, liver-only metastasis, lung-only metastasis, and multiple metastases were independent prognostic factors for PPS in PDAC patients following surgery. Moreover, multivariate analysis showed that CA19-9 was also an independent prognostic factor for PPS in these patients. In the Japanese Pancreas Society staging systems for pancreatic cancer, the para-aortic LN16 is categorized as a Group $3 \mathrm{LN}$ station. LN16 metastasis is considered indicative of distant metastasis and poor survival in PDAC (14). LN16 positivity is common in PDAC, and a standard lymphadenectomy of positive LN16 is helpful in elevating survival and has demonstrated the great impact of LN16 metastasis on PPS in PDAC patients (15). Compared with the other variables included in the present study, LN16 metastasis had the greatest impact on PPS, followed by liver-only metastasis, local recurrence, and multiple metastases. The distant genetic signatures of metastatic 


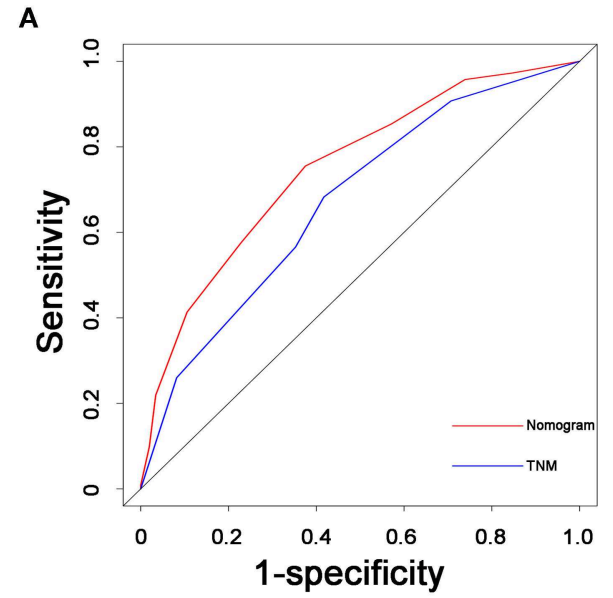

C

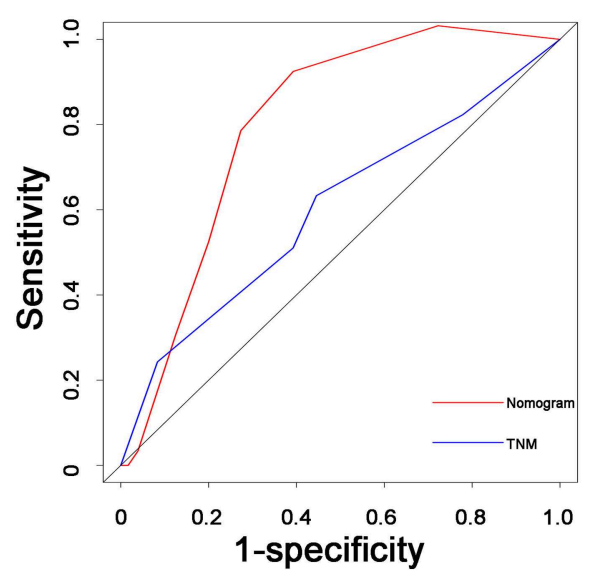

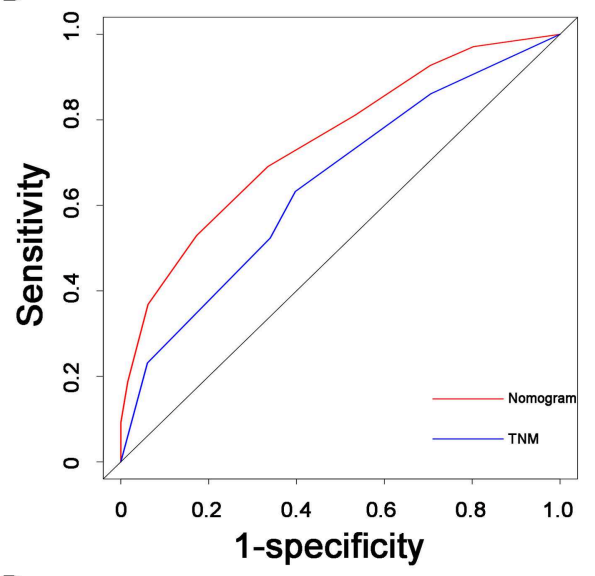

D

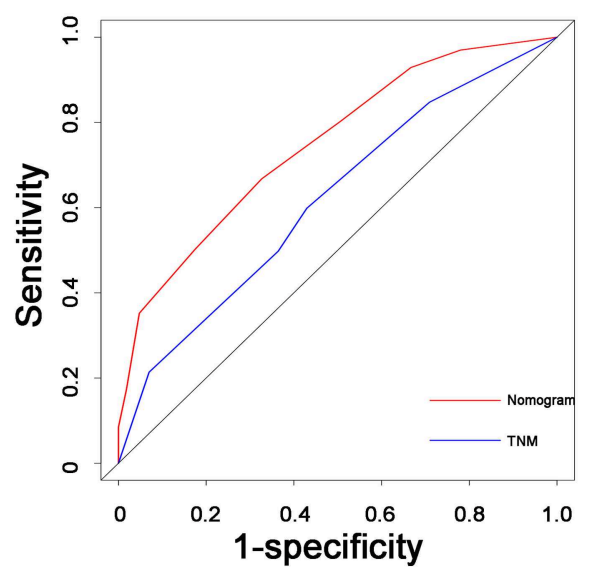

FIGURE 5 | Comparisons of receiver operating characteristic (ROC) curves of both the nomogram and TNM stage system for predicting 1- and 2-year PPS in the training cohort $(\mathbf{A}, \mathbf{B})$ and validation cohorts $\mathbf{( C , D )}$, respectively. TNM, tumor-node-metastasis; PPS, post-progression survival; PDAC, pancreatic ductal adenocarcinoma.

lesions might contribute to organ-specific metastases, and the exploration of their mechanisms could potentially illuminate personal therapeutic approaches.

Apart from the progression patterns, increased CA19-9 levels and tumor largeness were important characteristics of high tumor burden in PDAC, which indicated poor treatment response and early progression $(16,17)$. Poorly differentiated tumors indicated poor survival as well. A previous study indicated that poorly differentiated tumors release certain molecules, including epidermal growth factor and E-cadherin, which could enhance the development of distant metastases and shorten survival times (18). Compared with pathological tumor size, imaging-detected tumor size was more heavily weighted in the survival analysis and was considered an independent prognostic factor for PPS. The calculation or evaluation of the largest tumor size through image comparisons of different levels of tumors with a 1-mm interval was considered comprehensive and accurate. However, the measurement of the largest pathological tumor size was slightly more subjective, as it was nearly impossible to compare tumor sizes from each level of tumors. This may explain the greater role of imaging-detected tumor size compared with pathological size in predicting survival. In addition, the patients included in this study were from 2008 to 2018 and received no neoadjuvant chemotherapy. Following surgery, 142 patients received adjuvant chemotherapy and 160 patients did not receive adjuvant chemotherapy. Moreover, most of the patients were in the relatively early stages of PDAC (TNM I and II); this may explain the insignificance of chemotherapy in the survival analysis. Further evaluation of the prognostic value of chemotherapy in PDAC is needed.

A nomogram for PPS estimation was established based on these independent prognostic factors, which were selected by evaluating high-dimensional radiological and clinicopathological variables. Compared with traditional nomograms for survival prediction among PDAC patients, our nomogram relied on factors related to recurrence and more precisely indicated survival after tumor progression. Additionally, compared with the 8th TNM stage system, the presently developed predictive nomogram showed higher AUC and C-indexes values and 
stronger predictive power for PPS in both training and validation cohorts. The inclusion of specific indicators of progression patterns in addition to primary tumor characteristics ensured that the nomogram would display better discrimination power. Further, the relatively large cohort size of the present study could have made these results more generalizable than those from single-center studies with smaller numbers of patients. Physicians can use this nomogram to assess a variety of parameters with objectivity and precision and to distinguish between different subgroups of PPS among patients with PDAC following radical resection. Therefore, the presently established nomogram can be used as a practical tool to predict survival after tumor progression and has the potential for use in decision-making regarding the subsequent treatment of PDAC patients following surgery. Apart from the precise prediction of survival rates after tumor progression, the established nomogram had indicated several risk factors after surgery, including LN16, LN9, and LN14 metastases, poor tumor differentiation, and higher levels of CA19-9. Patients with these risk factors need to have adjuvant chemotherapy or radiochemotherapy as soon as possible after surgery to prolong survival. Additionally, when recurrence happens, this nomogram indicates that local recurrence and liver metastasis are more likely to lead to poorer survival, compared with lung metastasis. The additional special treatment for recurrence lesions or liver metastasis apart from the conventional chemotherapy, such as tumor ablation, may contribute to better survival for these patients.

The present study had several limitations. First, some variables were unavailable for this study, including specific treatment following surgery as well as the time period and regimen of chemotherapy. The inclusion of these variables could further support the feasibility of the nomogram for use with PDAC patients. Further, it was a limitation for the inclusion of local regression or metastases in that it neglected their time-related nature. Second, it is expected that more tumor progressions would be observed if the follow-up period were extended. Although all the patients were followed for more than 1 year, a longer follow-up period is needed for a more precise overview of tumor progression following surgery. Third, although neoadjuvant chemotherapy is an important factor that may have impacted prognosis, it was not included in the present analysis. Although good fitness was demonstrated for validation in the present study, we should recognize that bootstrapping is only helpful in reducing the overfit bias of the nomogram. More

\section{REFERENCES}

1. Bray F, Ferlay J, Soerjomataram I, Siegel RL, Torre LA, Jemal A. Global cancer statistics 2018: GLOBOCAN estimates of incidence and mortality worldwide for 36 cancers in 185 countries. CA Cancer J Clin. (2018) 68:394-424. doi: $10.3322 /$ caac. 21492

2. Rahib L, Smith BD, Aizenberg R, Rosenzweig AB, Fleshman JM, Matrisian LM. Projecting cancer incidence and deaths to 2030: the unexpected burden of thyroid, liver, and pancreas cancers in the United States. Cancer Res. (2014) 74:2913-21. doi: 10.1158/0008-5472.CAN-14-0155

3. Kamisawa T, Wood LD, Itoi T, Takaori K. Pancreatic cancer. Lancet. (2016) 388:73-85. doi: 10.1016/S0140-6736(16)00141-0 validations using large, independent cohorts are necessary for the validation of the present nomogram.

In conclusion, we compared the PPS of different progression patterns and established a nomogram to predict PPS in patients with postoperative recurrence of PDAC. Validation based on training and validation cohorts showed that this nomogram has great predictive power for survival. The exploration of risk factors and the establishment of this nomogram could illustrate new versions of personalized recurrence management for PDAC patients following surgery.

\section{DATA AVAILABILITY STATEMENT}

The raw data supporting the conclusions of this article will be made available by the authors, without undue reservation.

\section{ETHICS STATEMENT}

The studies involving human participants were reviewed and approved by the Institutional Review Board of Sun Yat-sen University Cancer Center. The patients/participants provided their written informed consent to participate in this study. Written informed consent was obtained from the individual(s) for the publication of any potentially identifiable images or data included in this article.

\section{AUTHOR CONTRIBUTIONS}

SL was responsible for the conception, design, and quality control of this study, reviewed and edited the manuscript. $\mathrm{CH}$, SS, and YZ performed the study selection, data extraction, and statistical analyses and were major contributors in writing the manuscript and contributed to the writing of the manuscript. $\mathrm{CH}$ and SS participated in study selection and statistical analyses. $\mathrm{CH}$, SS, YZ, and XL contributed in classification criteria discussion. All authors have read and approved the final version of the manuscript.

\section{FUNDING}

This work was supported by grants from the National Natural Science Funds (Nos. 81972299 and 81672390) and the National Key Research and Development Plan (No. 2017YFC0910002). 
7. He C, Huang X, Zhang Y, Cai Z, Lin X, Li S. A quantitative clinicopathological signature for predicting recurrence risk of pancreatic ductal adenocarcinoma after radical resection. Front Oncol. (2019) 9:1197. doi: 10.3389/fonc.2019.01197

8. Chang DK, Johns AL, Merrett ND, Gill AJ, Colvin EK, Scarlett CJ, et al. Margin clearance and outcome in resected pancreatic cancer. J Clin Oncol. (2009) 27:2855-62. doi: 10.1200/JCO.2008.20.5104

9. Gebauer F, Tachezy M, Vashist YK, Marx AH, Yekebas E, Izbicki JR, et al. Resection margin clearance in pancreatic cancer after implementation of the Leeds Pathology Protocol (LEEPP): clinically relevant or just academic? World J Surg. (2015) 39:493-9. doi: 10.1007/s00268-014-2808-4

10. He $\mathrm{CB}$, Lin XJ. Inflammation scores predict the survival of patients with hepatocellular carcinoma who were treated with transarterial chemoembolization and recombinant human type-5 adenovirus H101. PLoS ONE. (2017) 12:e0174769. doi: 10.1371/journal.pone.0174769

11. Groot VP, Gemenetzis G, Blair AB, Rivero-Soto RJ, Yu J, Javed AA, et al. Defining and predicting early recurrence in 957 patients with resected pancreatic ductal adenocarcinoma. Ann Surg. (2019) 269:1154-62. doi: 10.1097/SLA.0000000000002734

12. Kim YI, Song KB, Lee Y-J, Park K-M, Hwang DW, Lee JH, et al. Management of isolated recurrence after surgery for pancreatic adenocarcinoma. Br J Surg. (2019) 106:898-909. doi: 10.1002/bjs.11144

13. He W, Peng B, Tang Y, Yang J, Zheng Y, Qiu J, et al. Nomogram to predict survival of patients with recurrence of hepatocellular carcinoma after surgery. Clin Gastroenterol Hepatol. (2018) 16:756-64.e10. doi: 10.1016/j.cgh.2017.12.002

14. Kondo S. Japanese pancreas society staging systems for pancreatic cancer. In: Pancreatic Cancer. New York, NY: Springer (2010). p. 1035-50.
15. Liu C, Chen R, Chen Y, Fu D, Hong D, Hao J, et al. Should a standard lymphadenectomy during pancreatoduodenectomy exclude para-aortic lymph nodes for all cases of resectable pancreatic head cancer? A consensus statement by the Chinese Study Group for Pancreatic Cancer (CSPAC). Int J Oncol. (2015) 47:1512-6. doi: 10.3892/ijo.2015.3128

16. Shimizu T, Asakuma M, Tomioka A, Inoue Y, Hirokawa F, Hayashi M, et al. Span-1 and CA19-9 as predictors of early recurrence and lymph node metastasis for patients with invasive pancreatic cancer after pancreatectomy. Am Surg. (2018) 84:109-13. doi: 10.1177/000313481808400130

17. Liu L, Xu H, Wang W, Wu C, Chen Y, Yang J, et al. A preoperative serum signature of CEA+/CA125+/CA19-9 $\geq 1000 \mathrm{U} / \mathrm{mL}$ indicates poor outcome to pancreatectomy for pancreatic cancer. Int J Cancer. (2015) 136:2216-27. doi: 10.1002/ijc.29242

18. Shibata K, Matsumoto T, Yada K, Sasaki A, Ohta M, Kitano S. Factors predicting recurrence after resection of pancreatic ductal carcinoma. Pancreas. (2005) 31:69-73. doi: 10.1097/01.mpa.0000166998.04266.88

Conflict of Interest: The authors declare that the research was conducted in the absence of any commercial or financial relationships that could be construed as a potential conflict of interest.

Copyright (c) $2020 \mathrm{He}$, Sun, Zhang, Lin and Li. This is an open-access article distributed under the terms of the Creative Commons Attribution License (CC BY). The use, distribution or reproduction in other forums is permitted, provided the original author(s) and the copyright owner(s) are credited and that the original publication in this journal is cited, in accordance with accepted academic practice. No use, distribution or reproduction is permitted which does not comply with these terms. 